\title{
Impact of Print Bed Build Location on the Dimensional Accuracy and Surface Quality of Parts Printed by Multi Jet Fusion
}

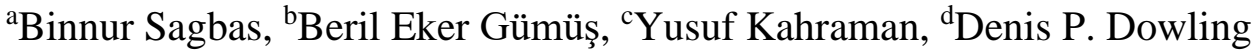
${ }^{a}$ Yildiz Technical University, Mechanical Engineering Department, 34349 Besiktas Istanbul, Turkey
bsagbas@gmail.com
${ }^{\text {b} Y i l d i z ~ T e c h n i c a l ~ U n i v e r s i t y ~ S c i e n c e ~ a n d ~ T e c h n o l o g y ~ R e s e a r c h ~ a n d ~ A p p l i c a t i o n ~ C e n t e r ~}$ Istanbul, Turkey beril.gumus@merklab.yildiz.edu.tr
'Turkish Airlines, Turkish Technic R\&D Center, 34912 Pendik, Istanbul, Turkey yus kah@hotmail.com

${ }^{\mathrm{d}}$ School of Mechanical and Materials Engineering, University College Dublin, Belfield, Dublin 4, Ireland.

\section{Abstract} denis.dowling@ucd.ie

\begin{abstract}
Multi Jet Fusion (MJF) is one of the newly developed additive manufacturing techniques, based on the use of powder bed fusion technology. It provides the opportunity to build up 3D, complex polymer geometries, without the need for support structures. This study evaluates the effect of build location across a $380 \times 284 \mathrm{~mm}^{2}$ build plate, on both the dimensional accuracy and surface quality of polyamide 12 (PA12) parts printed using the MJF technique. The cube test samples were printed at each of the four corners and the center of the build plate. Dimensional deviations were determined using optical metrology measurements, while surface deviations were measured using 2D tactile and 3D optical profilometers. The density and the degree of crystallinity of the samples were determined using the Archimedes method and Differential Scanning Calorimetry analysis, respectively. Moreover, the morphology of the internal polymer surfaces was evaluated using Scanning Electron Microscopy. It was concluded that while overall printed part dimensions and crystallinity homogeneity were relatively uniform across the build plate, some variations were observed. Parts printed in particular, closer to the front of the build plate exhibited higher porosity, higher surface roughness along with the highest level of geometry deviation, compared with the CAD drawing. This is likely to be associated with some non- uniformities in heating and cooling of the PA12 polymer parts across the build plate.
\end{abstract}

Keywords: Additive manufacturing, multi jet fusion, PA12, powder bed fusion, dimensional accuracy

\section{Introduction}

Multi Jet Fusion (MJF) is relatively newly developed, polymer based additive manufacturing (AM) processing technique, which was developed by Hewlett-Packard (HP) [1]. It belongs to the powder bed fusion (PBF) technology sub group, which also includes techniques such as 
binder jetting and selective laser sintering (SLS) [1,2]. Compared with SLS and binder jetting, the MJF technique is reported to offer the advantages of high productivity and low cost per unit volume, in terms of energy and material consumption [3]. SLS uses point based laser beam for sintering the polymer material across a line, while MJF uses IR lamps as the heat source, in order to fuse the polymer particles across the build plate [2,4]. The MJF equipment incorporates two stations, which are named as build unit and post processing unit. Two additive agents, known as the fusing and detailing agents are applied as a thin layer, over the polymer powder, in order to absorb IR radiation or to block it, respectively. The part printing steps are illustrated schematically in Figure 1. In the build unit, the first layer of polymer powder is spread over the build platform and it is heated to a near-sintering temperature. The fusing agent is precisely deposited on to the powder by inkjet nozzles, in accordance with 3D geometry of manufactured part, while the detailing agent is deposited near the edge of the part in order to act as a sintering inhibitor. The IR energy source passes over the powder on the build plate and sinters the areas where the fusing agent was deposited and leaves the rest of the powder unaltered. The process is repeated until all parts are fabricated [1,5]. The fusing agent contains carbon black, which makes the white polymer powder more light absorbing. The detailing agent lowers the temperature of the heated surface and create the boundary of built geometry from unfused powder. This detailing agent as its name suggests, helps the MJF process to more accurately print more geometrically complex features [6,7]. In comparison with corresponding methods, the MJF technique is reported to obtain higher dimensional accuracy by printing thirty million drops per second [8]. During the printing process, the 3D geometry is supported by the powder, so there is no need to the extra support structures, which can decrease the surface quality and dimensional accuracy of the manufactured part. After generation of the 3D geometry at the build station, cooling and unpacking processes are applied at the post processing unit. So the building up cycle continuing without any discontinuation, which clearly enhances processing efficiency [9]. This facilitates $80 \%$ powder re-use to the next printing process, thus facilitating greater processing sustainability [10].

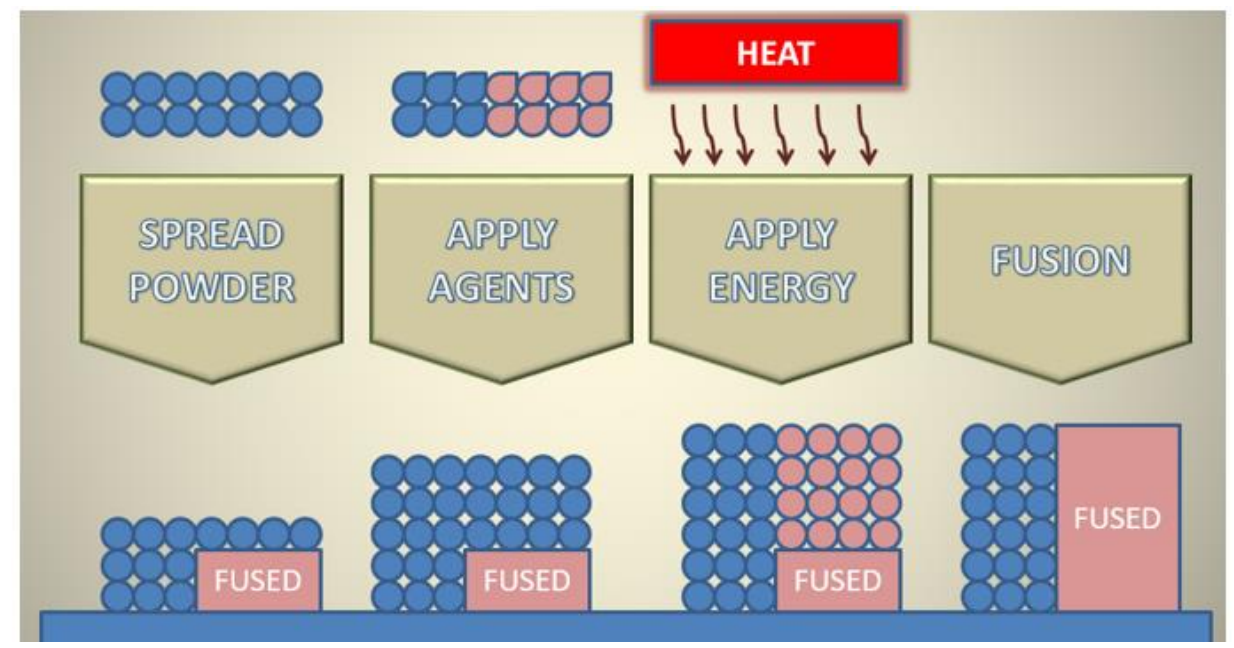

Figure 1. Schematic of the four steps involved in the printing of parts using the MJF system

The most widely used polymer with the MJF processing is polyamide (PA), which is a semicrystalline, thermoplastic polymer [1]. It is selected due to its relatively high mechanical strength, low melt viscosity [4], high impact resistance and low moisture absorbance. Due to its higher melting temperature, compared with that of crystallization, it has a wide "sintering window" [11], which provides slower crystallization and as a consequence reduction of distortions and residual stresses [3].The PA can be combined with glass beads, aluminum, silicon carbide, carbon nano tubes and nano fibers to produce composite structures, exhibiting 
enhanced mechanical performance [12]. Besides PA12 powder, glass bead added PA12 and PA11 powders are also suitable for MJF technology $[13,14]$

As MJF is a relatively new processing technology, there have been relatively few reports on the performance of the printed polymers or composites. Amongst these are two reports which have compared the performance of PA12 parts fabricated using the MJF and SLS techniques $[4,5]$. MJF provided 10 times faster printing speed and a superior surface quality compared with the SLS approach, however because of the detailing agent, the MJF technique typically exhibits lower densities and tensile strengths. Both these studies reported on the higher roughness's values obtained on the top face of the MJF printed parts. In the case of $\mathrm{Xu}$ et al.[4] it was speculated that this could be due to a lack of detailing agent on the top surface. In contrast the paper by Sillani et al. [5], speculated that this effect was due to a heat transfer mechanism i.e. the thermal bleeding problem of powder-bed fusion. These latter authors also highlighted the need to investigate the influence of part positioning on the build platform, which is the focus of the current study.

Riedelbauch et al. [6] focused on the reusability of PA12 powder in MJF process and determined aging effect on the mechanical properties of the samples manufactured in $\mathrm{Z}$ and $\mathrm{Y}$ direction. They concluded that thermal aging had no effect on the mechanical properties of the MJF parts. O'Connor et al.[7] investigated the effect of build orientation on the mechanical properties of MJF manufactured PA12 samples. It was reported that samples, manufactured in $\mathrm{X}, \mathrm{Y}, \mathrm{Z}$ orientations, exhibited isotropic behavior in terms of tensile strength, while flexural strength was highly affected by different build orientations. In a further study, O'Connor et al. [12] also investigated the effect of glass bead addition in to the PA12 powder and the authors reported that incorporation of glass beads increased the tensile and flexural modulus, while it decreased the tensile and flexural strength of the samples. Santos et al. [15] reported a comparative study about measurement techniques for additive manufacturing, by using a design-for-metrology approach. They used high speed sintering method which was similar to that of the MJF system, and it was concluded that there is a need for the development of measurement procedures and standards for the dimensional assessment of additive manufactured parts. In an investigation by Sağbaş [1], the effects of build orientation angle on both the dimensional accuracy and surface quality of MJF manufactured parts. The author reported that orientation angle has significant effect on the part properties, by decreasing the print top surface area. Higher surface deviations may occur on the flat top surfaces because of the amount of detailing agent or capillary effect, therefore it is recommended to avoid flat top surfaces in order to decrease these deviations [16] .

While MJF technology provides the opportunity to build up parts at different locations and geometric orientations at the same processing time, differences in orientation and layouts may cause parts to be exposed to different temperature gradients, which in-turn may alter the cooling rate, crystallinity, density etc. [17]. This may affect the resulting dimensional accuracy and mechanical properties of the printed parts. While there have been very few reports in the literature on the manufacturing efficiency of MJF processing, clearly some process optimisation is required related to build orientation, in order to optimize the resulting printed part performance. This study aims to address this issue, by evaluating the effect of build location on the MJF build plate, on dimensional accuracy and surface quality of the manufactured parts. The focus is on the evaluation of the processing efficiency of cubic parts, printed across the build plate. Establishing this print homogeneity is key to the wider adoption of this processing technology within manufacturing operations. 


\section{Materials and Methods}

\subsection{Manufacturing of the Samples}

This additive manufacturing study was carried out using a HP 3D Multi Jet Fusion 4200 printer, which has a build plate with dimensions of $380 \times 284 \times 380 \mathrm{~mm}^{3}$. Cube samples with dimensions of 20x20x20 mm $\mathrm{mm}^{3}$, were printed using HP 3D High Reusability PA12 powder, at the five positions on to the build plate as illustrated in Figure 2. The printing study was repeated on three separate occasions using the same processing conditions and part lay-out on the print bed. For the individual cubes, each face was named according to its direction and position. The front faces of the cubes are numbered according to their positions such as 1, 2, 3, 4 and 5 (Figure 2). The faces, which are opposite to the numbered front faces were named as "Y". Bottom faces of the cubes were named as "B", while the top faces (last to be printed) were named as " $Z$ ". The faces which are towards the outer side of the Cube- 1 and Cube- 2 and inner side of the Cube- 4 and Cube-3 were named as "X" while their opposites were named as "O". Build plate orientations and names of the cube faces are illustrated in Figure 2.

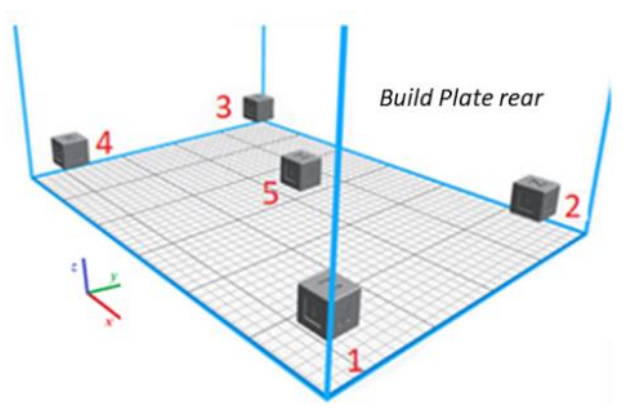

a

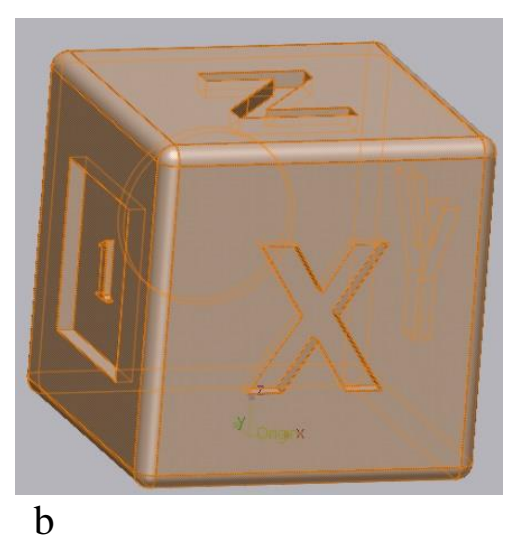

b

Figure 2. Build orientations of the cubes on to the build plate, with cubes 1 and 4 placed at the front of the plate (a), Examples of the letters printed on each face of the printed cubes (b).

The PA12 powder was sourced from HP, its material data sheet reported that it has a melting point of $187^{\circ} \mathrm{C}$, while powder bulk density and print density were $0.425 \mathrm{~g} / \mathrm{cm}^{3}$ and $1.01 \mathrm{~g} / \mathrm{cm}^{3}$ respectively. The mean diameter of the PA12 powder was reported to be approximately $60 \mu \mathrm{m}$ [14]. Since it provides a good compromise between dimensional accuracy, mechanical properties, surface roughness and printing speed, the samples were built up in "balanced print mode" and "natural cooling" were applied [16]. After printing, there was a delay of approx. 50 minutes to facilitate the cooling of the built parts to approximately $45^{\circ} \mathrm{C}$. Once cooled to this temperature, the build station containing the print bed, could be brought to the post-processing station. Because the printed parts are encapsulated by powder, support structures are not necessary in the MJF process. It is therefore only required to remove excess powder from around the parts and they were then glass bead blasting (under 5 bar pressure). Before the surface measurements, the samples were washed in deionized water using an ultrasonic bath for about 20 minutes and then dried under ambient conditions. Scanning Electron Microscope (SEM) images of HP 3D High Reusability PA12 powder and glass beads can be seen in Figure 3. 

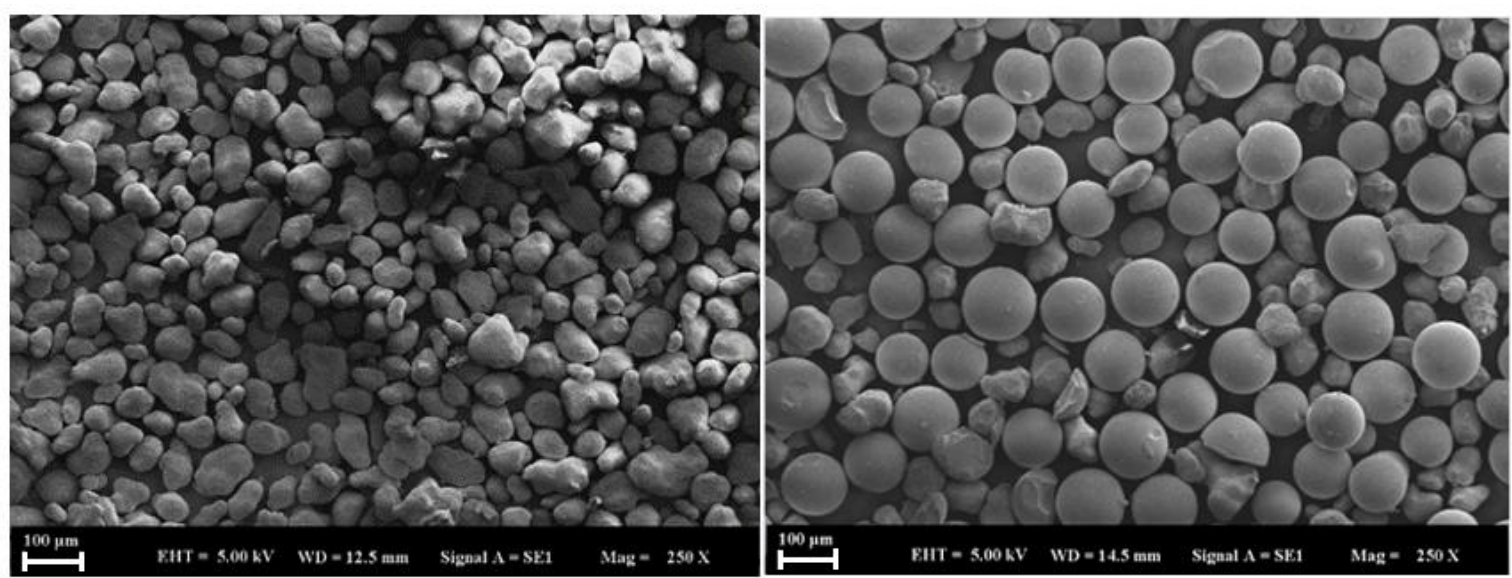

Figure 3. SEM images of PA12 powder (left) and glass bead used for blasting (right) (scale bar $100 \mu \mathrm{m})$

\subsection{Dimensional Measurements}

Dimensional and geometrical deviations, caused by shrinkage and capillarity, of the cubes were inspected using a Solutionix Rexcon CS2+ 3D optical scanner. Cubes were fixed onto the rotating table of the system and scanned from 10 different positions for obtaining measured geometry. The CAD data of the cubes were used as reference nominal geometry for comparison with measured geometry. These two geometries were matched and evaluated by Geomagic Control X.

\subsection{Surface Roughness Measurements}

2D and 3D surface roughness measurements were taken by Brucker Dektak XT tactile mechanical profilometer and AEP Nanomap 1000WLI optical profilometer respectively. Five repeated measurements were taken on each surface of the cubes, so for 6 faces of a cube 30 roughness value were recorded. Arithmetic mean values of the results were calculated and compared with each surface on a cube and surfaces of the other cubes. Effect of build location on surface roughness were evaluated.

\subsection{Density Measurements}

Densities of the each sample were defined according to the Archimedes principle by Precisa XB220A electronic balance with precision scale $0.0001 \mathrm{~g}$. Since the densities of the PA12 is lower than $1 \mathrm{~g} / \mathrm{cm}^{3}$, water was unsuitable for measurement, therefore ethyl alcohol was used for these density measurements.

\subsection{Scanning Electron Microscope Analysis}

For inspecting the cube inner structures, for porosity or other defects, the cubes were cut close to their centers. Zeiss EVO LS10 Scanning Electron Microscope were used for taking the images after Au coating of the sample surfaces.

\subsection{Differential Scanning Calorimetry (DSC) Analysis}

DSC measurements of the specimens $(8 \pm 1 \mathrm{mg}, \mathrm{n}=5)$ were performed using a Perkin Elmer Diamond DSC 8230 (Perkin-Elmer, Waltham, MA) under nitrogen atmosphere (flow rate:50 $\mathrm{mL} / \mathrm{min}$ ). The obtained data was analyzed by using Pyris Software. The specimens were tested 
with the heating rate of $10^{\circ} \mathrm{C} / \mathrm{min}$ within the range of $20-300{ }^{\circ} \mathrm{C}$. The heat capacity was obtained using the half-height tangent method. The melting enthalpy was obtained by integrating the fusion peak. The CI (crystalline index), $\chi_{c}$, of the samples were calculated with the following equation:

$\chi_{c}=\frac{\Delta H_{m}^{i}}{\Delta H_{m}^{0}} \times 100$

Here, $\Delta H_{m}^{i}\left(\mathrm{~J} \mathrm{~g}^{-1}\right)$ refers to the melting enthalpy of the current sample and $\Delta H_{m}^{0}\left(\mathrm{~J}_{\mathrm{g}} \mathrm{g}^{-1}\right)$ is the

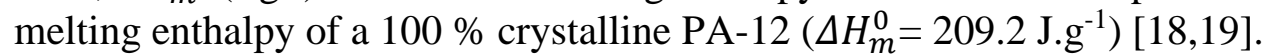

\section{Results and Discussions}

\subsection{Dimensional Measurements}

Deviations from the nominal geometry were defined by comparing CAD data of nominal geometry and measured data of manufactured geometry of the cubes. Measurements were taken from 20 different points on each surface of the cube and in total 120 measurements were recorded for all the surfaces of each cube. Measurement locations on the cube surface are illustrated in Figure 4. Arithmetical mean values and standard deviations of the measurement results were calculated for all three repeated print experiments. Maximum positive and negative dimensional deviations and their average values for each surface of the five cube samples are reported in Table 1. These values provide an indication of how the printed cube location either exhibits higher or lower dimensions, compared with the CAD drawing of the part.

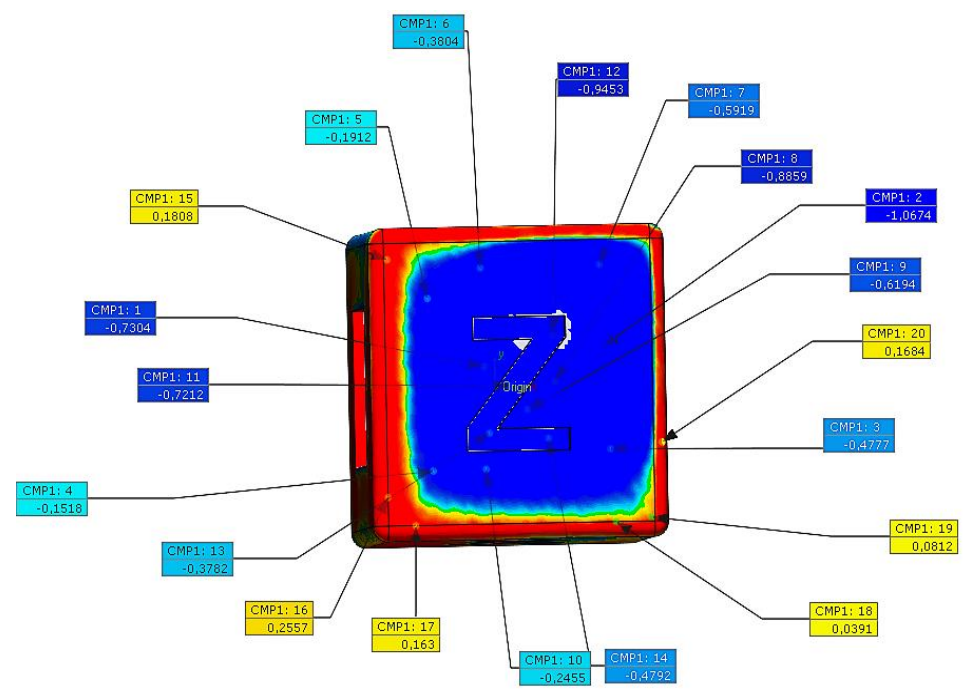

Figure 3. Demonstration of the location of where dimensional deviations measurements were obtained on the surface of the cubes.

Table 1. Deviations from the nominal dimensions (mm) - Reported values are the mean of three print experiment.

\begin{tabular}{|c|c|c|c|c|c|c|}
\hline Surfaces & & CUBE-1 & CUBE-2 & CUBE-3 & CUBE-4 & CUBE-5 \\
\hline \multirow{4}{*}{$\mathbf{X}$} & Min. & 0.003 & -0.013 & -0.047 & -0.012 & -0.036 \\
\hline & Max. & 0.209 & 0.061 & 0.041 & 0.121 & 0.093 \\
\hline & Avg. & 0.07 & 0.021 & -0.003 & 0.032 & 0.021 \\
\hline & SD & 0.062 & 0.018 & 0.024 & 0.039 & 0.033 \\
\hline $\mathbf{Y}$ & Min. & -0.003 & -0.074 & -0.118 & -0.087 & -0.047 \\
\hline
\end{tabular}




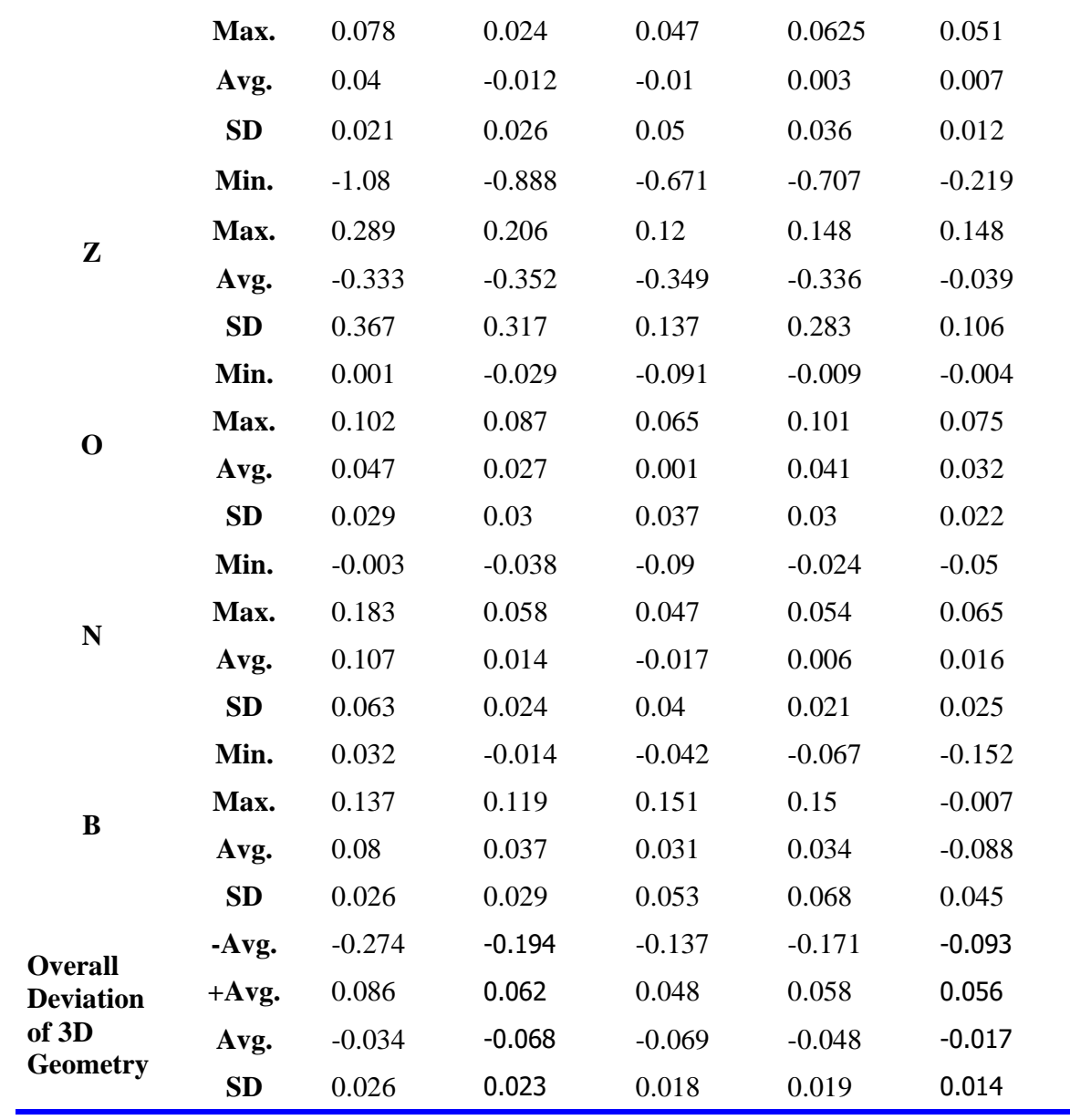

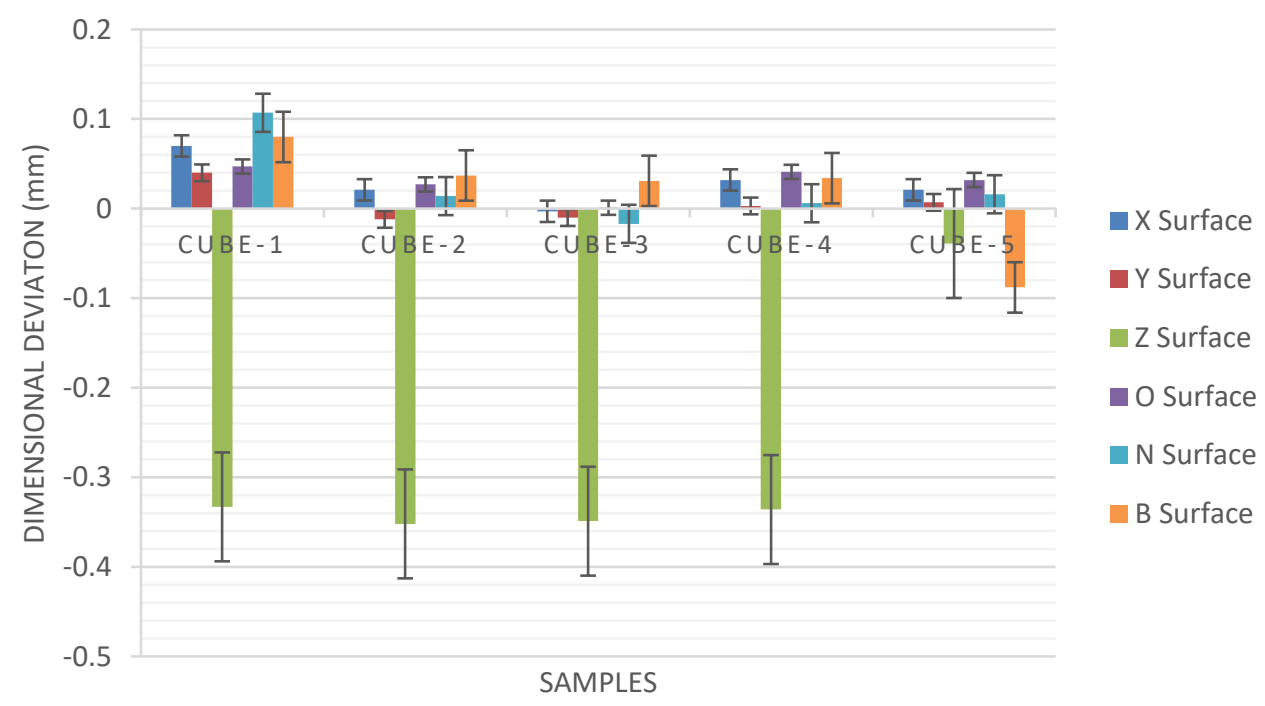

Figure 4. Graphical representation of the dimensional deviations on each surface of the cubes

Although the overall dimensional deviations were low, with mean dimensional deviations of between -17 to $-69 \mu \mathrm{m}$, obtained for the five cubes ( 3 print runs), the average positive and negative deviations were relatively high such as -274 to $86 \mu \mathrm{m}$. The highest negative dimensional deviation was obtained for the $\mathrm{Z}$ surface of all the cube samples, with the exception of Cube-5, which were located on to the center of build plate. For this, the small maximum 
negative dimensional deviation was measured on the B surface of Cube-5. For Cubes 1 to 4, the deviations recorded on the B surface were positive. Minimum dimensional deviation was measured on Cube-5 surfaces. The color scale given in Figure 6, helps to graphically demonstrate the measured geometry deviations of the cubes. Note the blue color for the $\mathrm{Z}$ surface, highlighting the negative deviation from the CAD values. It is clear from Table 1 and Figure 5 that the highest deviations between the CAD and measured cube dimensions were obtained for Cube-1, which was located on the front right of the print bed. Negative deviation was recorded for $\mathrm{Z}$ surface of the Cube-1, while other surfaces exhibited positive deviations. Deviations of $\mathrm{Y}$ and $\mathrm{O}$ surfaces, located towards the inner side of the build plate, were lower than the deviations obtained for the $\mathrm{N}$ and $\mathrm{X}$ surfaces, located towards the outer side of the build chamber. Positive deviation was recorded for the B surface also. Similar characteristics were recorded for Cube- 4 with lower deviation amounts. Deviation characteristics on Cube- 2 and Cube-3, located at the back wall side of the build chamber, were also similar in terms of $\mathrm{Y}, \mathrm{Z}$ and B surfaces. Finally as demonstrated in Figure 5, no significant deviations were observed on the $\mathrm{X}$ and $\mathrm{O}$ surfaces of Cube-3, for which the deviations in both the positive and negative directions, were approximately the same.
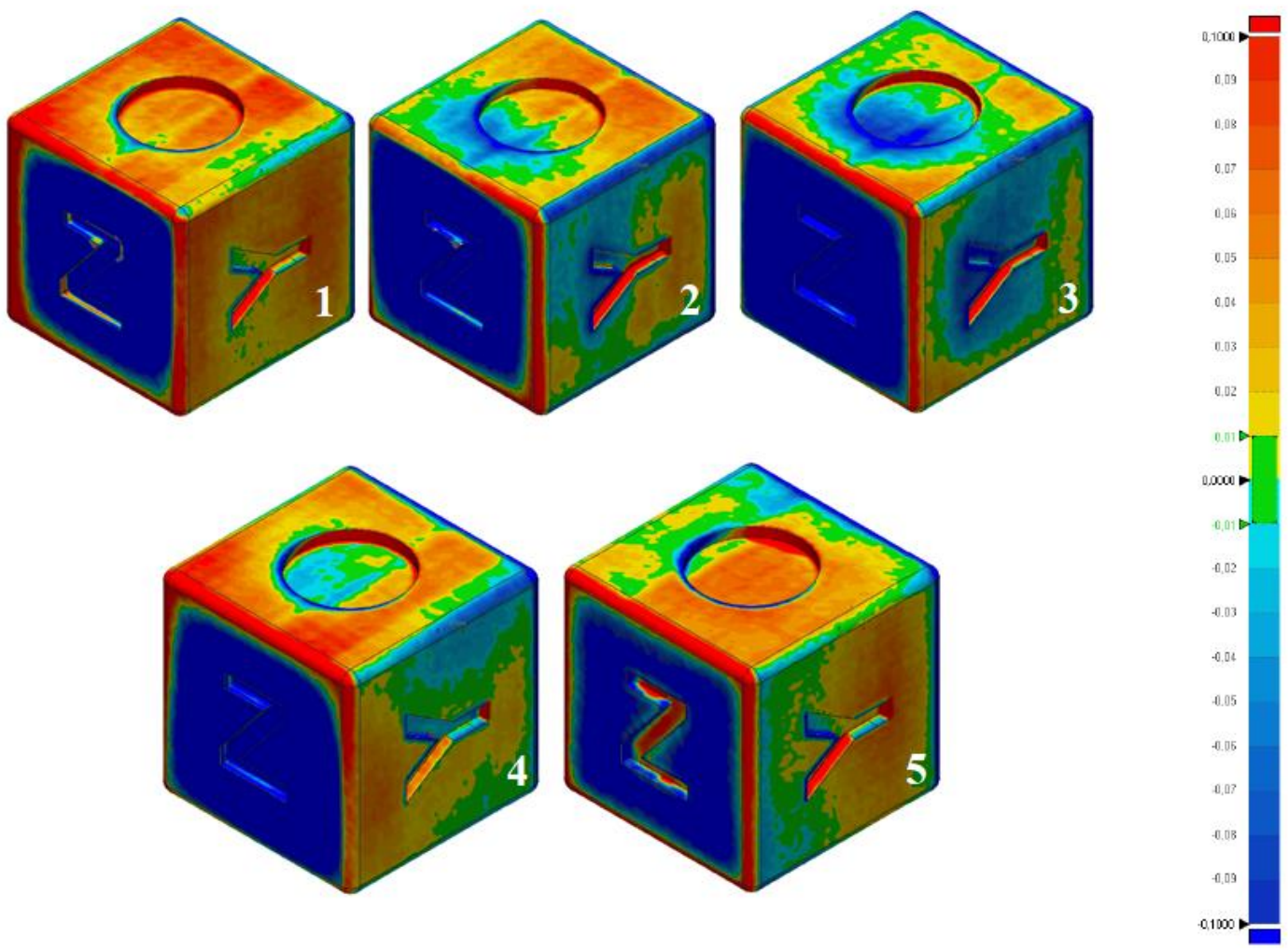

Figure 5. 3D representation of total dimensional deviation obtained for cubes 1 to 5 , all printed in the same print run. Note the consistently negative deviation for the $\mathrm{Z}$ face of all 5 cubes. The scale bar on the right hand side is for the range -100 to $+100 \mu \mathrm{m}$.

A more detailed analysis was carried out for the $\mathrm{Z}$ surface at the five print locations, as this was the surface exhibiting the highest deviation from the CAD drawing. Measurements were obtained across the cross sectional frame, on the center line of the cubes (Figure 7). The average deviations for the $\mathrm{Z}$ surface of all 5 cubes are given in Table 2. It is clear from the table that the maximum deviation is observed for Cube 1 and the minimum for Cube 5 . 

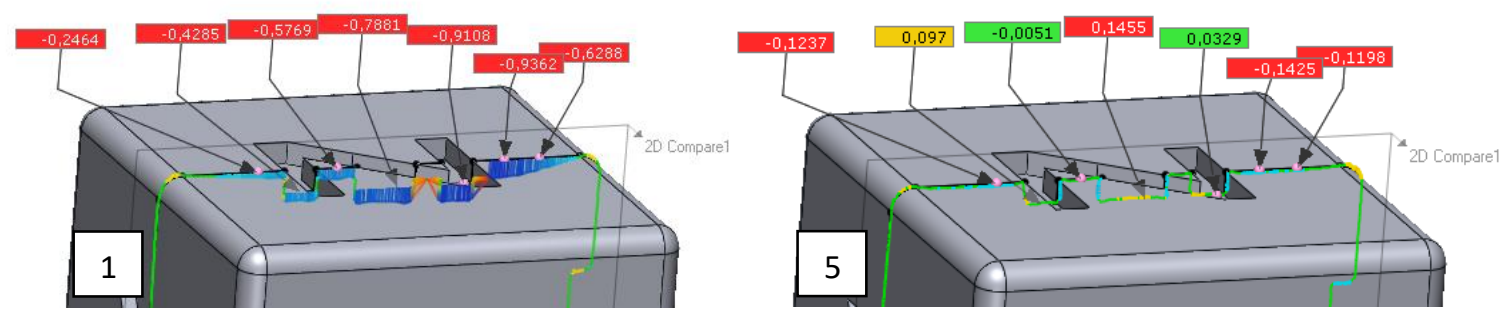

Figure 6. Locations where deviations were measured on the $\mathrm{Z}$ surface. The minimum deviations were obtained for Cube- 1 and the maximum for Cube-5.

Table 2. Deviations of the $\mathrm{Z}$ surfaces of all the cubes (average of 3 print runs)

\begin{tabular}{llllll}
\hline Deviations $(\mathbf{m m})$ & Cube-1 & Cube-2 & Cube-3 & Cube-4 & Cube-5 \\
\hline Max.deviation & -0.247 & -0.378 & -0.227 & -0.178 & 0.141 \\
Min.deviation & -0.923 & -0.858 & -0.668 & -0.721 & -0.132 \\
Average deviaiton & -0.653 & -0.632 & -0.513 & -0.532 & -0.017 \\
\hline
\end{tabular}

Unlike from laser based powder bed fusion systems, all area of the build layer of the part is fused in a single pass in the MJF system. While this yields higher productivity, it may result in thermal gradients, especially for the large print area. When the detailing agent is sprayed over the top surface of the built part, the water solvent evaporates, resulting in a decrease in the polymer temperature $[5,6]$. The level of the heat transfer is unlikely to be equal at all region of the part [5]. The rate of temperature decrease would be faster in the edge regions where heat transfer is higher to the surrounding polymer grains, it is likely to be slower in the center of the part, which will benefit from the effect of the temperature of the previous layers. The generated thermal gradients may in-turn cause non-uniform cooling and so uneven shrinkage along the build surface [16]. In order to evaluate this, the temperature variation across the build chamber was measured. It was not possible to obtain in-line measurements of the build plate temperature during building and cooling processes, however the MJF system facilitates the monitoring of the temperature distribution based on thermal camera measurements within the building layer, as it is being printed. A typical top view of the temperature distribution of the build chamber is given in the Figure 8. It demonstrated that there are some non-uniformities in the temperature distribution of the built layer. Just to highlight that dimensional deviations may be as a result of the non-uniform temperature gradients during both building and cooling processes, which could not be monitored with the current monitoring system.
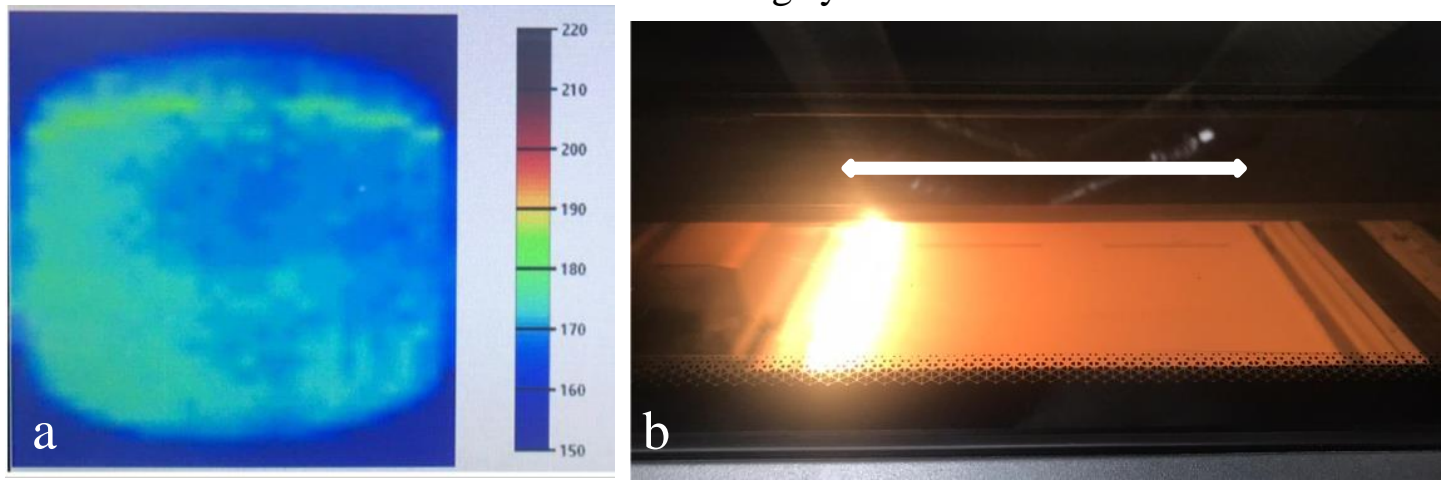

Figure 7. a) Temperature variations within the $380 \times 284 \mathrm{~mm}$ the MJF print bed. For this scan the recorded bed temperature was in the range approximately $170-185^{\circ} \mathrm{C}$. b) Photograph of 
the MJF process showing the heating effect of the IR lamps, along with an arrow indicating the reciprocting movement of these lamps.

Moreover, capillarity also causes deviations on the top surface of the built part. This is generated through the interaction of the fused powder and agents onto the top surface of the bed [20]. The fused layer behaves like a fluid and tends to raise up to the side borders, which causes subsidence at the center of the surface as explained in Ref. [20]. Therefore, in order to minimize the impact of thermal variations, parts should be positioned on the bed in a manner which would minimize the top surface area, so as to decrease the effect of thermal gradients and capillarity effect on dimensional deviations, as highlighted by other authors $[1,21]$.

In summary, it was concluded from this study that the largest dimensional deviations were obtained for the cube $\mathrm{Z}$ surfaces, which is located at the upper surface of the cube (last to be printed). Cubes located at four of the five locations on the print bed exhibited higher deviations at this face. The exception was Cube-5, which was located at the center of build plate. Nonuniform cooling may cause uneven shrinkage which typically exhibited itself as concave features at the center of the $\mathrm{Z}$ surfaces, with upward at the edges. A factor influencing this is the differences in the rate of the heat loss across the surface. Heat transfer rate is higher to the surrounding from the edge regions of the surface while it is slower towards to the center of the surface with the effect of the temperature of the previous layer. This factor is more effective at the location of the side borders of the print bed. Therefore, Cube- 5 being furthest from the edge of the print bed on all but its top surface, exhibited printed dimensional closest to the CAD value.

\subsection{Surface Texture Analysis Results}

The results of both 2D profile [22] and 3D area surface roughness [23] measurements are reported in Tables 4 and 5 respectively, for each surface of the 5 sample cubes.

Table 4. 2D Profile Surface Roughness Measurements of the cubes in $\mu \mathrm{m}$ (average of 3 print runs)

\begin{tabular}{|c|c|c|c|c|c|c|c|c|c|c|c|c|}
\hline \multirow{3}{*}{$\begin{array}{c}\text { Cube } \\
\text { Number }\end{array}$} & \multicolumn{12}{|c|}{ Surfaces } \\
\hline & \multicolumn{2}{|c|}{$\mathbf{Z}$} & \multicolumn{2}{|c|}{$\mathbf{X}$} & \multicolumn{2}{|c|}{$\mathbf{Y}$} & \multicolumn{2}{|c|}{ O } & \multicolumn{2}{|c|}{$\mathbf{N}$} & \multicolumn{2}{|c|}{ B } \\
\hline & Ra & $\mathbf{R q}$ & Ra & $\mathrm{Rq}$ & Ra & Rq & $\mathbf{R a}$ & Rq & Ra & Rq & Ra & $\mathbf{R q}$ \\
\hline Cube-1 & 23.46 & 28.21 & 16.78 & 20.60 & 14.76 & 17.98 & 17.74 & 22.20 & 9.37 & 11.98 & 13.78 & 16.74 \\
\hline Cube-2 & 17.36 & 21.44 & 14.47 & 18.40 & 11.17 & 13.49 & 13.58 & 17.62 & 11.66 & 14.78 & 12.22 & 15.05 \\
\hline Cube-3 & 20.10 & 23.92 & 12.89 & 15.67 & 10.59 & 13.33 & 13.77 & 17.60 & 11.69 & 15.17 & 11.56 & 13.90 \\
\hline Cube-4 & 16.38 & 20.25 & 12.79 & 16.10 & 9.76 & 12.74 & 15.66 & 19.21 & 11.79 & 14.55 & 9.87 & 11.96 \\
\hline Cube-5 & 13.69 & 17.05 & 12.34 & 15.04 & 12.46 & 14.96 & 10.31 & 12.54 & 10.23 & 13.10 & 7.18 & 8.66 \\
\hline
\end{tabular}


Table 5. 3D Arial Surface Roughness Measurement Results in $\mu \mathrm{m}$ (average of 3 print runs)

\begin{tabular}{|c|c|c|c|c|c|c|c|c|c|c|c|c|}
\hline \multirow{3}{*}{$\begin{array}{c}\text { Cube } \\
\text { Number }\end{array}$} & \multicolumn{12}{|c|}{ Surfaces } \\
\hline & \multicolumn{2}{|c|}{$\mathbf{Z}$} & \multicolumn{2}{|c|}{$\mathbf{X}$} & \multicolumn{2}{|c|}{$\mathbf{Y}$} & \multicolumn{2}{|c|}{ O } & \multicolumn{2}{|c|}{$\mathbf{N}$} & \multicolumn{2}{|c|}{ B } \\
\hline & Sa & $\mathrm{Sq}$ & Sa & $\mathbf{S q}$ & Sa & $\mathrm{Sq}$ & Sa & $\mathrm{Sq}$ & Sa & $\mathrm{Sq}$ & Sa & Sq \\
\hline Cube-1 & 15.33 & 19.36 & 10.90 & 12.36 & 11.85 & 14.80 & 10.10 & 12.72 & 8.68 & 10.97 & 9.59 & 11.72 \\
\hline Cube-2 & 12.36 & 17.65 & 6.63 & 8.92 & 8.48 & 11.87 & 8.98 & 11.80 & 10.23 & 13.03 & 8.51 & 11.33 \\
\hline Cube-3 & 8.71 & 12.21 & 8.61 & 11.47 & 8.75 & 11.46 & 8.60 & 11.17 & 7.96 & 10.86 & 8.15 & 11.27 \\
\hline Cube-4 & 8.80 & 10.75 & 8.16 & 10.55 & 6.61 & 8.72 & 8.19 & 10.40 & 7.90 & 9.78 & 7.16 & 9.15 \\
\hline Cube-5 & 8.44 & 10.73 & 7.40 & 9.74 & 5.62 & 7.66 & 6.38 & 8.62 & 7.08 & 9.30 & 6.04 & 8.09 \\
\hline
\end{tabular}

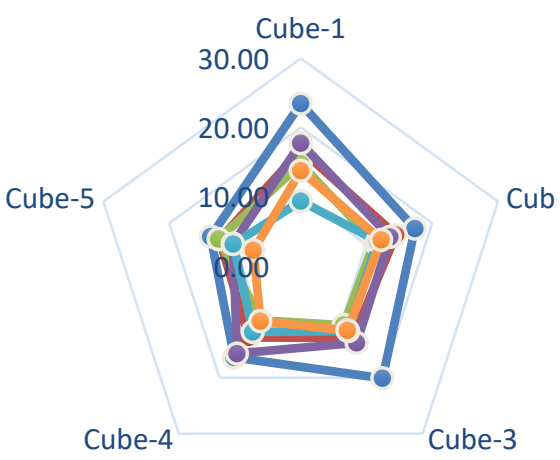

- Z Surface $=0$ X Surface $=$ Y Surface

-O Surface $=$ N Surface $=B$ Surface

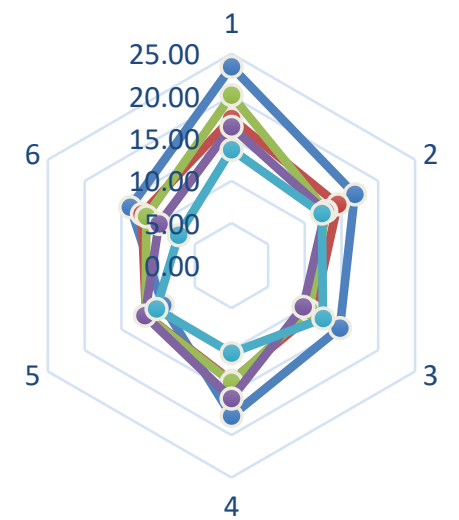

-Cube-1 -0 Cube-2 $\Rightarrow$ Cube-3

-Cube-4 Cube-5

Figure 8. 2D arithmatic mean surface roughness (Ra in $\mu \mathrm{m}$ ) values of each of the surfaces of the individual cube samples.

It is clear from the Figure 9 that, 2D arithmetic mean surface roughness was highest on the top surface " $Z$ " of all the cubes, while the base surface "B" exhibits the lower roughness values. The enhanced level of roughness on the top surfaces was also reported by previous authors of MJF parts, as detailed in the introduction section [4,5]. The more detailed investigation in the current study demonstrates the typically concave features at the center of the $\mathrm{Z}$ surfaces, with upward at the edges, possibly due to the non-homogeneous cooling of this surface, which is influenced by a combination of evaporation of the water solvent from the detailing agent (detailed section 3.1), as well as the more rapid rate of heat loss for this cube surface which is located closest to the print bed edge. The surface deviations were measured by 3D optical profilometer and the images of the $\mathrm{Z}$ surfaces were captured from the same region of the cubes (Figure 10). It is clear from the topographical images given in this figure that arising from the non-uniform shrinkage some warpages at this top surface of the samples, is observed. As before the higher deviations and roughness values were recorded for these $\mathrm{Z}$ surfaces. The lowest roughness values and roughness differences between the surfaces were recorded for the Cube5 printed at the center of the bed, while the highest values and differences were measured for the Cube-1. These results agree with dimensional deviations results detailed earlier. It is clear that, variations in temperature gradients across the build plate, affected cubes differently at different locations on the print bed, resulting in surface texture deviations, particularly on the $\mathrm{Z}$ surfaces [5]. 

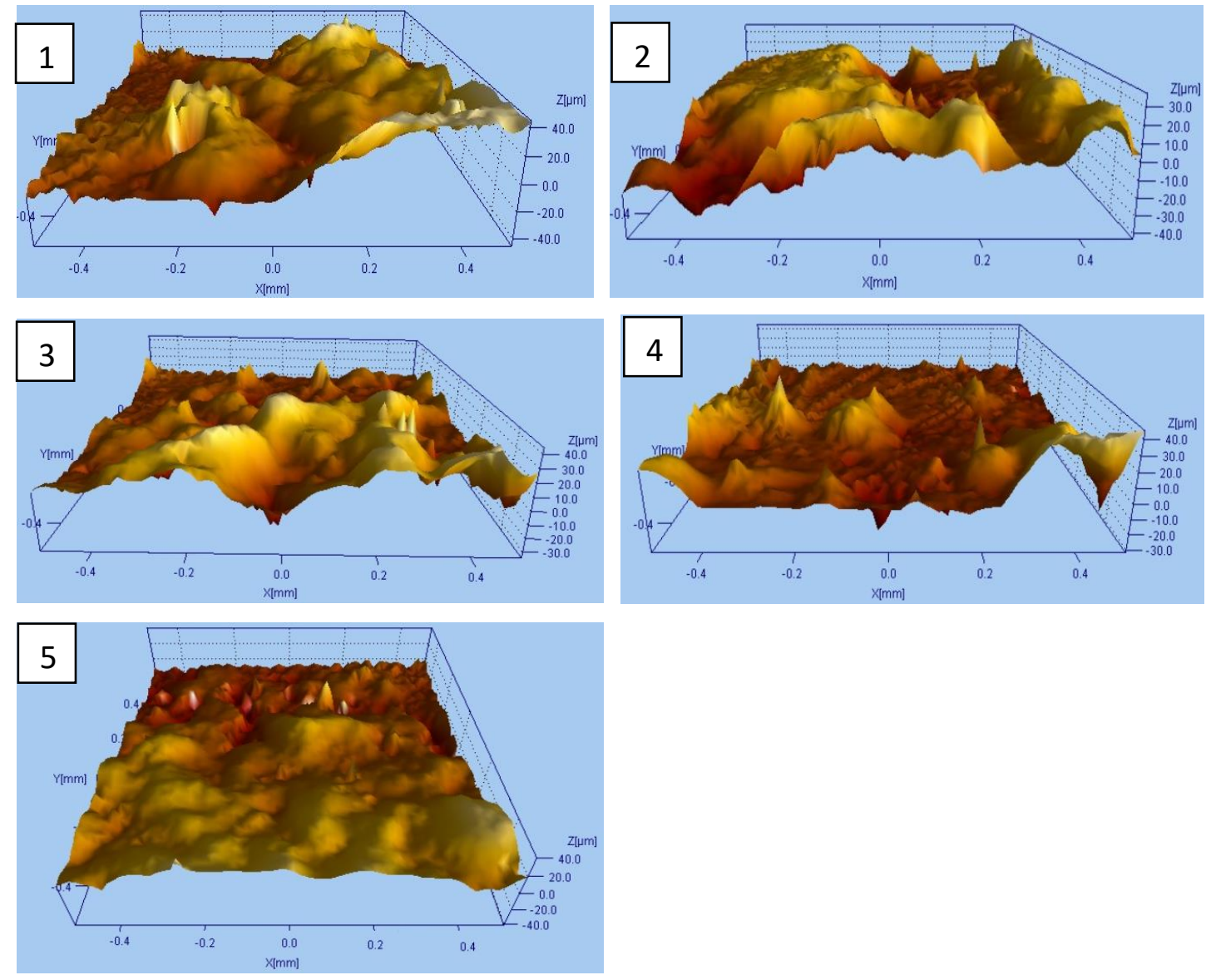

Figure 9. 3D optical profilometer images of the "Z" surfaces of the Cube-1 and Cube-5.

\subsection{Density and Crystallinity Measurement Results}

The density values of the cubes, measured by Archimedes' method, were found to be similar to each other and were in the range 0.982 to $0.991 \mathrm{~g} / \mathrm{cm}^{3}$. The results of the DSC analysis and calculated degrees of the crystallinity can be seen in the Figure 11. The melting enthalpy was defined by integrating the melting peak area and percentage of crystallinity was calculated using Equation 1. 


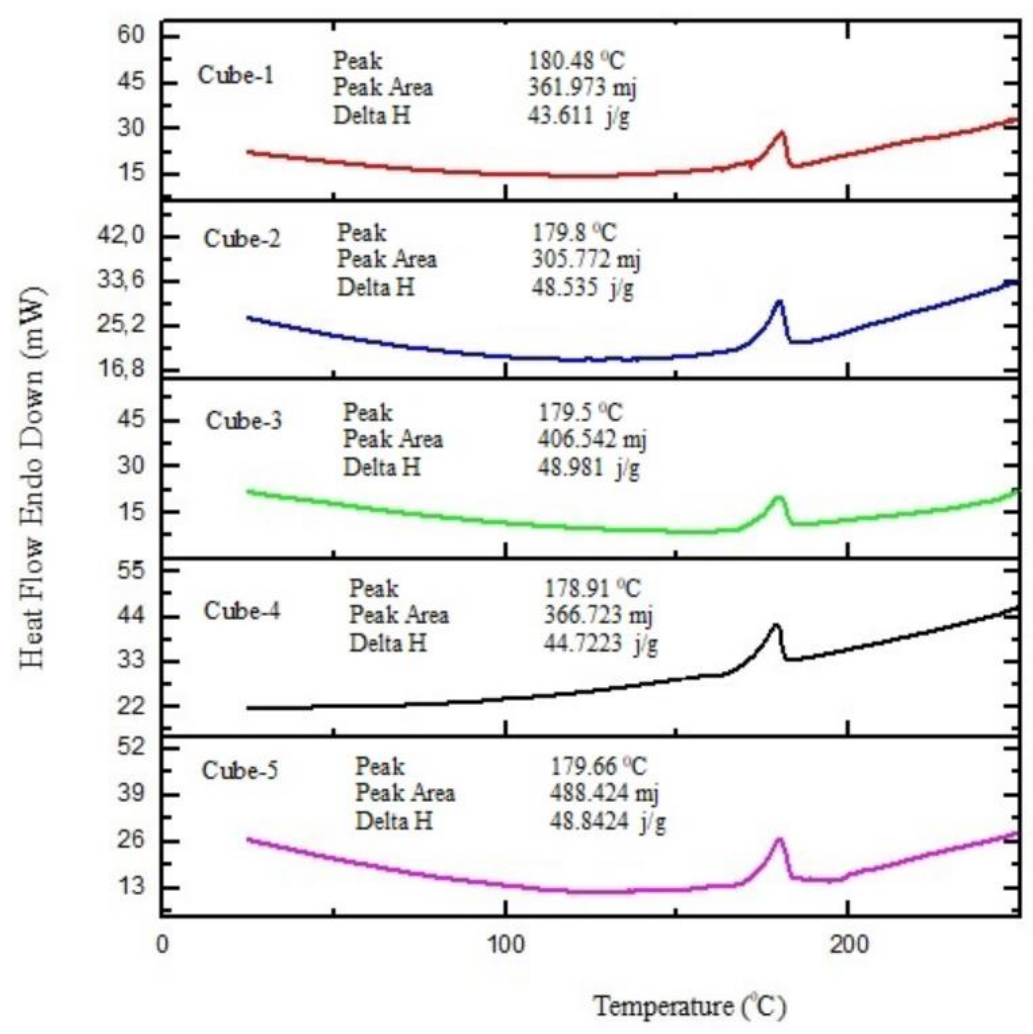

a)

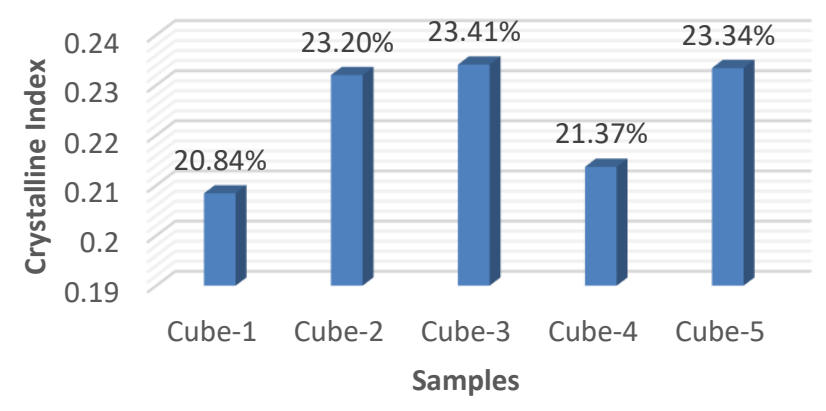

b)

Figure 10. DSC measurement results (a) and calculated degree of crystallinity (b)

The lowest crystallinity was recorded for Cubes 1 and 4, which as shown in Figure 2, were located at the front border of the build chamber. These were also the cubes exhibiting the highest dimensional deviations. Cubes 2, 3 and 5 exhibited both higher crystallinity and lower levels of geometry expansion. The highest crystallinity was calculated as $23.41 \%$, while typical values reported in the literature are 30\% for MJF parts [4], with 24\% for SLS manufactured PA12 parts [4]. The degree of crystallinity results obtained in this study, were in accordance with the density values.

Density and crystallinity are important parameters for determining porosity and dimensional deviations of the additive manufactured polymer parts. While a semicrystalline thermoplastic polymer is heated it dilates and dimensional expansion occurs. If it is cooled from this temperature, it tends to come back to its initial dimension. But depending on the cooling condition and shrinkage, the polymer does not revert to its original dimensions. Both cooling 
rate and thermal gradient affect crystallization index, in addition to shrinkage, which in turn impact on the dimensional accuracy of the printed parts [24].

\subsection{SEM Analysis Results}

The internal structures of the cubes were examined using SEM, based on the examination of areas near the center of the cubes. There were no visible pores in Cubes 3 and 5, while pore like structures were detected in Cube 1, 2 and 4 (Figure 12). The SEM images of Cubes 1 and 5 , visibly demonstrating the differences in the level of porosity obtained. These results clearly correlated with the degree of crystallinity of the cubes and again indicated the impact of nonuniform temperature gradients and cooling rates, at different locations on the build plate.
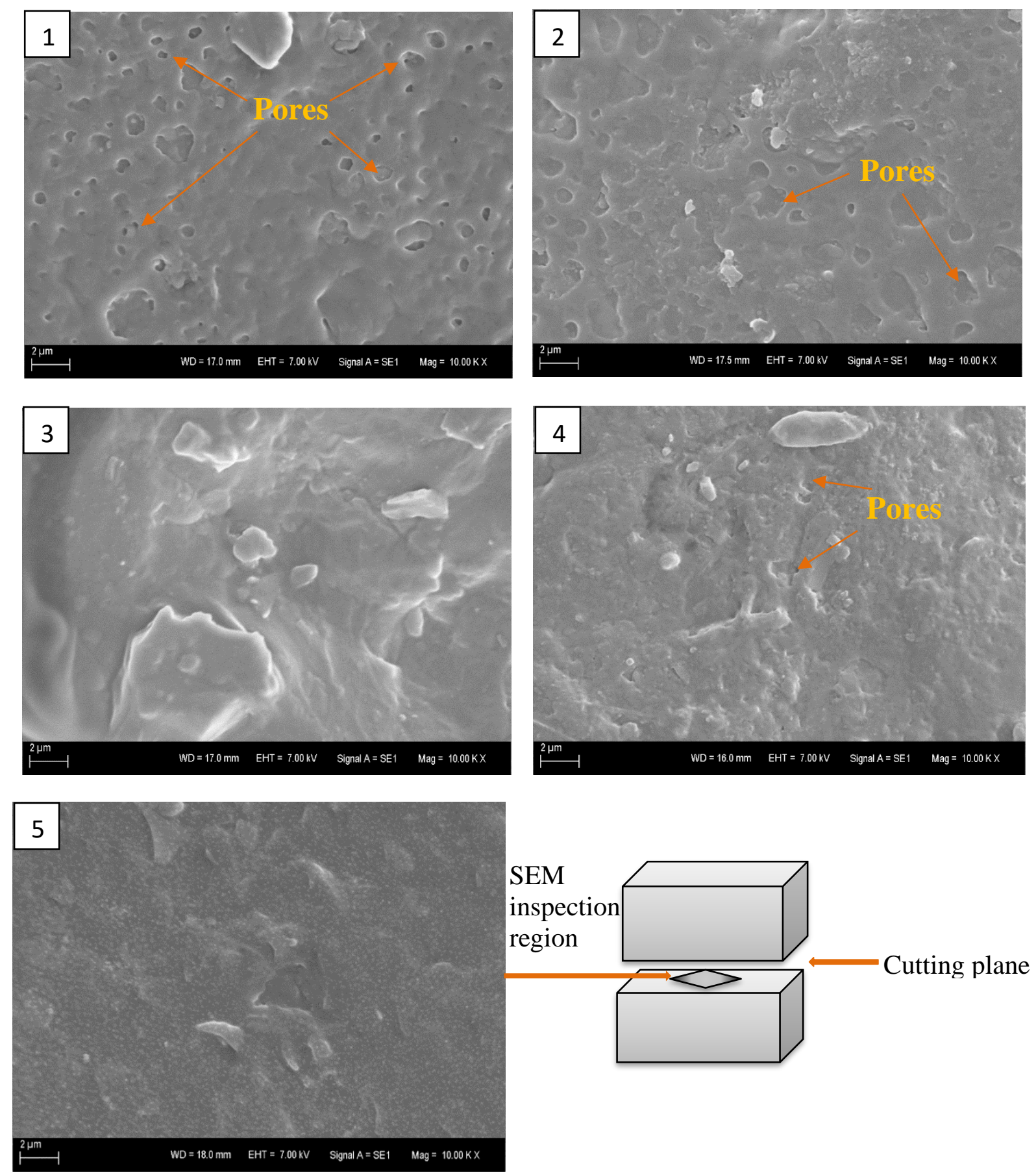
Figure 11. SEM images showing examples of the morphology of the inner structure obtained for each of the five Cube samples, at the location indicated by the schematic diagram

\section{Conclusion}

The objective of this study was to evaluate the effect of print bed build location on the dimensional accuracy and surface quality of the MJF manufactured PA12 parts. It is concluded from this study that -

- Based on an examination of both the dimensional deviation and roughness of the printed cubes printed at the 5 locations on the print bed, that the surface quality of the printed cubes was high, with a mean overall dimensional deviation with CAD values, across the five $20 \times 20 \times 20 \mathrm{~mm}^{3}$ cubes ( 3 print runs), were in the range of -17 to $-69 \mu \mathrm{m}$.

- The largest dimensional deviations were obtained for the cube $\mathrm{Z}$ surfaces, which is located at the upper surface of the cube (last to be printed). A factor influencing this is the differences in the rate of the heat loss across the surface. Heat transfer rate is higher to the surrounding from the edge regions of the surface while it is slower towards to the center of the surface with the effect of the temperature of the previous layer. This is the likely reason that Cube-5, which is located furthest from the edge of the print bed on all but its top surface, exhibited printed dimensional closest to the CAD value.

- As for the dimensional deviations the higher deviations and roughness values were recorded for the $\mathrm{Z}$ surfaces. As highlighted by previous authors higher surface deviations may occur on the flat top surfaces because of the amount of detailing agent or capillary effect [16]. An additional factor influencing roughness highlighted in this study is temperature distribution. The lowest roughness values and roughness differences between the surfaces were recorded for the Cube-5, while the highest values and differences were measured for the Cube-1. The latter was located at the front right of the build plate. A possible explanation for this is the presence of temperature gradients across the build plate, which will clearly influence both the level of thermal energy that the polymer is exposed too during heating, in addition to the subsequent cooling rate.

- DSC measurements of polymer crystallinity indicated that parts printed close to the back of the plate exhibited values of approx. $21 \%$, while those printed in the center and the front of the build plate were approx. 23\%. These results correlated with dimensional deviations with highest values for the cubes at the back of the bed.

Overall it is concluded from this study that while PA12 cube parts printed using the MJF across the $380 \times 284 \times 380 \mathrm{~mm}^{3}$ print bed, yielded relatively homogeneous part crystallinity and dimensional uniformity. The dimensional, roughness and crystallinity deviations were largely associated between parts printed towards the front of the bed and those at the rear of the bed. This is likely to be associated with a small variation of the temperature distribution between these two regions of the print bed.

\section{Conflict of Interest}

The authors declare no conflict of interest.

\section{Acknowledgement}

In this study PA12 cube samples were manufactured by MJF, by Form Additive Manufacturing and Robotics Inc.-Turkey. Dimensional measurements were supported by Mayis Tasarim- 
Turkey. The authors would like to thank them for their support. This work is partially supported by the I-Form Advanced Manufacturing Research Centre (Grant Number 16/RC/3872).

\section{References}

[1] B. Sağbaş, Effect of Orientation Angle on Surface Quality and Dimensional Accuracy of Functional Parts Manufactured by Multi Jet Fusion Technology, Eur. Mech. Sci. 4 (2020) 47-52. https://doi.org/10.26701/ems.678901.

[2] C.N. Kelly, J. Francovich, S. Julmi, D. Safranski, R.E. Guldberg, H.J. Maier, K. Gall, Fatigue behavior of As-built selective laser melted titanium scaffolds with sheet-based gyroid microarchitecture for bone tissue engineering, Acta Biomater. (2019). https://doi.org/10.1016/j.actbio.2019.05.046.

[3] S. Yuan, F. Shen, C.K. Chua, K. Zhou, Polymeric composites for powder-based additive manufacturing: Materials and applications, Prog. Polym. Sci. (2019). https://doi.org/10.1016/j.progpolymsci.2018.11.001.

[4] Z. Xu, Y. Wang, D. Wu, K.P. Ananth, J. Bai, The process and performance comparison of polyamide 12 manufactured by multi jet fusion and selective laser sintering, J. Manuf. Process. (2019). https://doi.org/10.1016/j.jmapro.2019.07.014.

[5] F. Sillani, R.G. Kleijnen, M. Vetterli, M. Schmid, K. Wegener, Selective laser sintering and multi jet fusion: Process-induced modification of the raw materials and analyses of parts performance, Addit. Manuf. $27 \quad$ (2019) 32-41. https://doi.org/10.1016/J.ADDMA.2019.02.004.

[6] J. Riedelbauch, D. Rietzel, G. Witt, Analysis of material aging and the influence on the mechanical properties of polyamide 12 in the Multi Jet Fusion process, Addit. Manuf. 27 (2019) 259-266. https://doi.org/10.1016/J.ADDMA.2019.03.002.

[7] H.J. O'Connor, A.N. Dickson, D.P. Dowling, Evaluation of the mechanical performance of polymer parts fabricated using a production scale multi jet fusion printing process, Addit. Manuf. 22 (2018) 381-387. https://doi.org/10.1016/J.ADDMA.2018.05.035.

[8] S. Morales-Planas, J. Minguella-Canela, J. Lluma-Fuentes, J.A. Travieso-Rodriguez, A.A. García-Granada, Multi Jet Fusion PA12 manufacturing parameters for watertightness, strength and tolerances, Materials (Basel). (2018). https://doi.org/10.3390/ma11081472.

[9] C.A. Chatham, T.E. Long, C.B. Williams, Progress in Polymer Science A review of the process physics and material screening methods for polymer powder bed fusion additive manufacturing, 93 (2019) 68-95.

[10] M. Mele, G. Campana, G.L. Monti, A Decision Method to Improve the Sustainability of Post Processing in Multi Jet Fusion Additive Manufacturing, in: Procedia Manuf., 2020. https://doi.org/10.1016/j.promfg.2020.02.101.

[11] M. Schmid, A. Amado, K. Wegener, Polymer powders for selective laser sintering (SLS), in: AIP Conf. Proc., 2015. https://doi.org/10.1063/1.4918516.

[12] H.J. O' Connor, D.P. Dowling, Comparison between the properties of polyamide 12 and glass bead filled polyamide 12 using the multi jet fusion printing process, Addit. Manuf. 31 (2020) 100961. https://doi.org/10.1016/J.ADDMA.2019.100961.

[13] M. Galati, F. Calignano, S. Defanti, L. Denti, Disclosing the build-up mechanisms of multi jet fusion: Experimental insight into the characteristics of starting materials and 
finished parts, J. Manuf. Process. (2020). https://doi.org/10.1016/j.jmapro.2020.06.029.

[14] HP 3D Printing materials, (November 2019), (n.d.). https://www8.hp.com/h20195/v2/GetDocument.aspx?docname=4AA7-1533ENA (accessed August 6, 2020).

[15] V.M. Rivas Santos, A. Thompson, D. Sims-Waterhouse, I. Maskery, P. Woolliams, R. Leach, Design and characterisation of an additive manufacturing benchmarking artefact following a design-for-metrology approach, Addit. Manuf. (2020). https://doi.org/10.1016/j.addma.2019.100964.

[16] HP MJF Handbook- General Recommendations for Printing Processes, (March 2019), (n.d.). https://enable.hp.com/us-en-3dprint-mjfhandbook (accessed August 6, 2020).

[17] C.A. Chatham, T.E. Long, C.B. Williams, A review of the process physics and material screening methods for polymer powder bed fusion additive manufacturing, Prog. Polym. Sci. (2019). https://doi.org/10.1016/j.progpolymsci.2019.03.003.

[18] Y. Zhou, S. Xi, Y. Huang, M. Kong, Q. Yang, G. Li, Preparation of near-spherical PA12 particles for selective laser sintering via Plateau-Rayleigh instability of molten fibers, Mater. Des. (2020). https://doi.org/10.1016/j.matdes.2020.108578.

[19] S.D. Tohidi, N. Dencheva, Z. Denchev, A.M. Rocha, B. Engesser, Development and characterization of single polymer composites prepared by compression molding of polyamide 6 empty microcapsules and novel woven textile structures, Mater. Today Commun. (2020). https://doi.org/10.1016/j.mtcomm.2020.100912.

[20] M. Mele, G. Campana, G.L. Monti, Modelling of the capillarity effect in Multi Jet Fusion $\begin{array}{lllll}\text { technology, } & \text { Addit. } & \text { Manuf. } & 30 & \text { (2019) }\end{array}$ https://doi.org/10.1016/J.ADDMA.2019.100879.

[21] HP MJF Handbook-Design for Aesthetics, (n.d.). https://img04.en25.com/Web/HPIncMSCRMProd/\%7B0976e370-05a5-4560-b6d067f079b185c7\%7D_3_1_3.pdf?elqTrackId=94ac8a0d568549538c667a7d44015b2b\&el qaid=2120\&elqat=2 (accessed August 6, 2020).

[22] ISO 4287, Geometrical Product Specifications (GPS) -- Surface texture: Profile method -- Terms, definitions and surface texture parameters, Int. Organ. Stand. (1997).

[23] ISO 25178 Series, Geometric Product Specifications (GPS) - Surface texture: areal, Int. Organ. Stand. (2010).

[24] L. Benedetti, B. Brulé, N. Decreamer, K.E. Evans, O. Ghita, Shrinkage behaviour of semi-crystalline polymers in laser sintering: PEKK and PA12, Mater. Des. (2019). https://doi.org/10.1016/j.matdes.2019.107906. 